\title{
First evidence of serotoninergic components in the nervous system of the monogenean Chimaericola leptogaster (Chimaericolidae, Polyopisthocotylea), a gill parasite of the relict holocephalan fish
}

\author{
Natalya V. Mochalova ${ }^{1}$, Nadezhda B. Terenina ${ }^{1}$, Larisa G. Poddubnaya ${ }^{2}$, Valery A. Yashin ${ }^{3}$, Andrei V. Kuchin \\ and Natalya D. Kreshchenko ${ }^{3}$ \\ ${ }^{1}$ Centre of Parasitology, A.N. Severtsov Institute of Ecology and Evolution, Russian Academy of Sciences, Moscow, Russia; \\ ${ }^{2}$ I.D. Papanin Institute for Biology of Inland Waters, Russian Academy of Sciences, Borok, Yaroslavl Province, Russia; \\ ${ }^{3}$ Institute of Cell Biophysics, Russian Academy of Sciences, Pushchino, Moscow Region, Russia
}

\begin{abstract}
The localisation and distribution of the serotoninergic nerve elements was studied for the first time in the flatworm Chimaericola leptogaster (Leuckart, 1830) using immunocytochemical methodology and confocal laser scanning microscopy. The musculature was investigated by histochemical staining of actin filaments; scanning electron microscopy was used to identify the sensory structures on the worm's surface. Uniciliated, bi-ciliated and multiciliated sensory endings have been described on the worm's surface. The morphological data demonstrate the presence of circular, longitudinal and diagonal muscles that comprise the musculature of $C$. leptogaster in the anterior, median and posterior body regions. Well-developed radial and circular muscle fibres were also observed surrounding the genital pore, two vaginae and in clumps of the haptor. The study revealed the presence of biogenic amine, serotonin, in the central and peripheral nervous systems of $C$. leptogaster: in the neurons and fibres of the cephalic ganglia and ventral nerve cord, in the innervation of reproductive system compartments. The localised sites of the serotoninergic elements point to important roles of serotonin in monogenean reproductive processes and, possibly, in the regulation of muscle function.
\end{abstract}

Keywords: serotonin, reproductive system, muscle system, haptor, Platyhelminthes

The class of parasitic flatworms, the Monogenea, is a key group in debates on the controversial question of the origin of the Neodermata because the nervous system in each class of flatworms is an evolutionary conservative structure and knowledge of its morphological characteristics is valuable in investigating the processes of macroevolution (Raikova et al. 2017). The general topography of the monogenean nervous system (NS), studied by visualisation of cholinesterase activity, revealed the typical orthogonal pattern of NS with three pairs of longitudinal nerve cords and transverse commissures (McKay et al. 1991, Timofeeva and Kotikova, 1995, Halton et al. 1998).

According to these authors the characteristic features of the monogenean NS are twofold: the presence of the anterior 'brain', which innervates the anterior body organs, and the existence of the posterior 'brain', which innervates a haptor (or attachment organ). The literature data indicate that the ventral nerve cords in monogeneans are the most developed: they send their fibres to the reproductive organs and digestive tract as well as to the attachment organs (Halton and Jennings 1964, Halton and
Morris 1969, Venkatanarsaiah and Kulkarni 1980, Halton et al. 1987, 1998).

The cholinergic, peptidergic and aminergic elements in the nervous system of a number of monopisthocotylean and polyopisthocotylean monogeneans were identified using immunocytochemical and histochemical methods (Arafa et al. 2007, Petrov et al. 2016, Hodová et al. 2018).

Some reports claim that cholinergic and peptidergic nerve elements are dominant in the monogenean central nervous system, whereas the peripheral nervous system consists mostly of serotoninergic nerve fibres (Maule et al. 1990a,b, Cable et al. 1996). Scarce immunocytochemical data on the localisation of serotonin in the monogenean nervous system were supported by Terenina and Gustafsson (2003) using the biochemical isolation of serotonin in tissue homogenates of Polystoma integerrimum (Froelich, 1791). It is assumed that, in monogeneans, the neurosubstances studied acted as neurotransmitters, taking part in the parasite's body-muscle contractions, innervating the adhesive organs, the gut and the reproductive structures, as well as participating in sensory function (Maule et al. 1989, 1990a,b, El-Naggar et al. 2004, 2007).

Address for correspondence: Nadezhda B. Terenina, Centre of Parasitology, A.N. Severtsov Institute of Ecology and Evolution of Russian Academy of Sciences, Leninsky Prosp. 33, Moscow, Russia. E-mail: terenina_n@mail.ru 

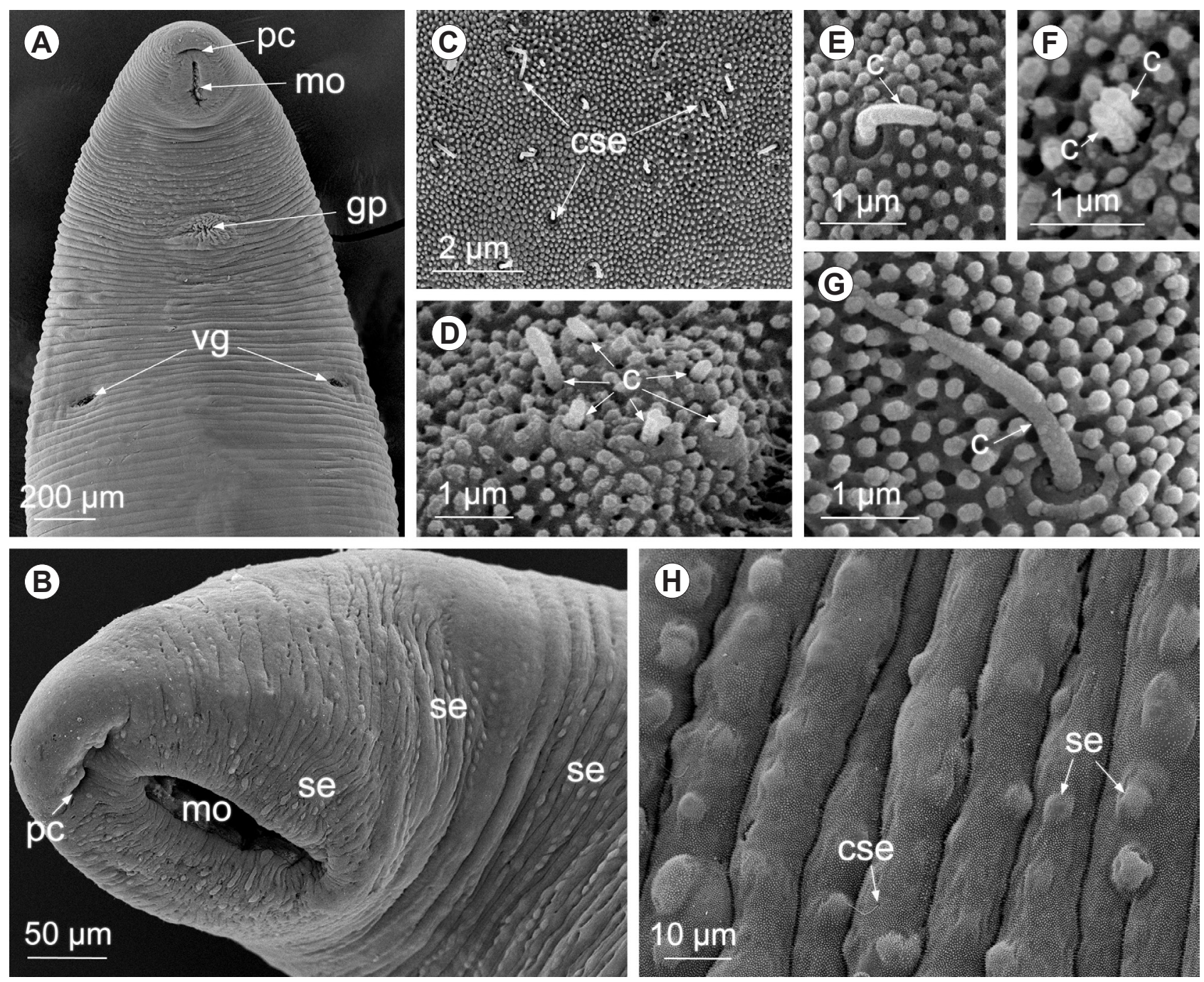

Fig. 1. Scanning electron microscopical images of the anteroventral body surface of Chimaericola leptogaster (Leuckart, 1830). A-ventral view of the forebody, showing mouth opening, genital pore and two vaginae; $\mathbf{B}$ - view of the anteroventral body note numerous ciliated and non-ciliated sensory endings; $\mathbf{C}$ - ciliated sensory endings around mouth opening; $\mathbf{D}$ - papillate multiciliated sensory ending, showing cilia of different length; $\mathbf{E}$ - sensory ending with middle-sized cilium; $\mathbf{F}$ - biciliated sensory ending; $\mathbf{G}$ - sensory ending with long cilium; $\mathbf{H}$ - ventral portion of the forebody showing numerous ciliated and non-ciliated sensory endings. Abbreviations: c - cilia; cse - ciliated sensory endings; gp - genital pore; mo - mouth opening; pc - preoral crevice; se - non-ciliated sensory endings; vg - vagina.

Chimaericola leptogaster (Leuckart, 1830) is parasite that lives on the gills of the deep-water rabbitfish, Chimaera monstrosa Linnaeus, a fish that belongs to the ancient and relict group of holocephalan fish, whose ancestors diverged from sharks roughly 400 million years ago (Inoue et al. 2010). There is no information on the nervous system of the chimaerocolid monogenean in the available literature.

In the present study, the classical neurotransmitter, serotonin (5-hydroxytryptamine, 5-HT), was identified for the first time in the nervous system of $C$. leptogaster using the immunocytochemical method and confocal laser scanning microscopy (CLSM). Preliminary information on the musculature morphology of C. leptogaster was obtained by staining of actin filaments with a fluorophore-conjugated phalloidin (Wahlberg 1998). Scanning electron microscopy (SEM) was used to identify sensory structures.

\section{MATERIALS AND METHODS}

\section{Monogenean and the fixation procedure}

Adult specimens of Chimaericola leptogaster were obtained from the gills of Chimaera monstrosa caught at the depth of 500-750 meters in the Norwegian Sea off Tromsø, Norway. They were caught from the RV 'Johan Ruud' which belongs to Tromsø University, using a deep-water trawl. Live worms were fixed in $4 \%$ paraformaldehyde (PFA) in $0.1 \mathrm{M}$ phosphate buffer (PBS, Sigma, Ronkonkoma, NY, USA) at $4^{\circ} \mathrm{C}$ and $\mathrm{pH}$ 7.4. For storage, the worms were transferred to the PBS buffer with $10 \%$ sucrose (Sigma) and kept until they were stained.

\section{Immunocytochemistry}

Whole mounts and cryostat sections of worms were stained with rabbit anti-5-HT (Immunostar, Hudson, WI, USA) (1 : 500) primary antibodies in PBS containing $1 \%(\mathrm{v} / \mathrm{v})$ Triton X-100 

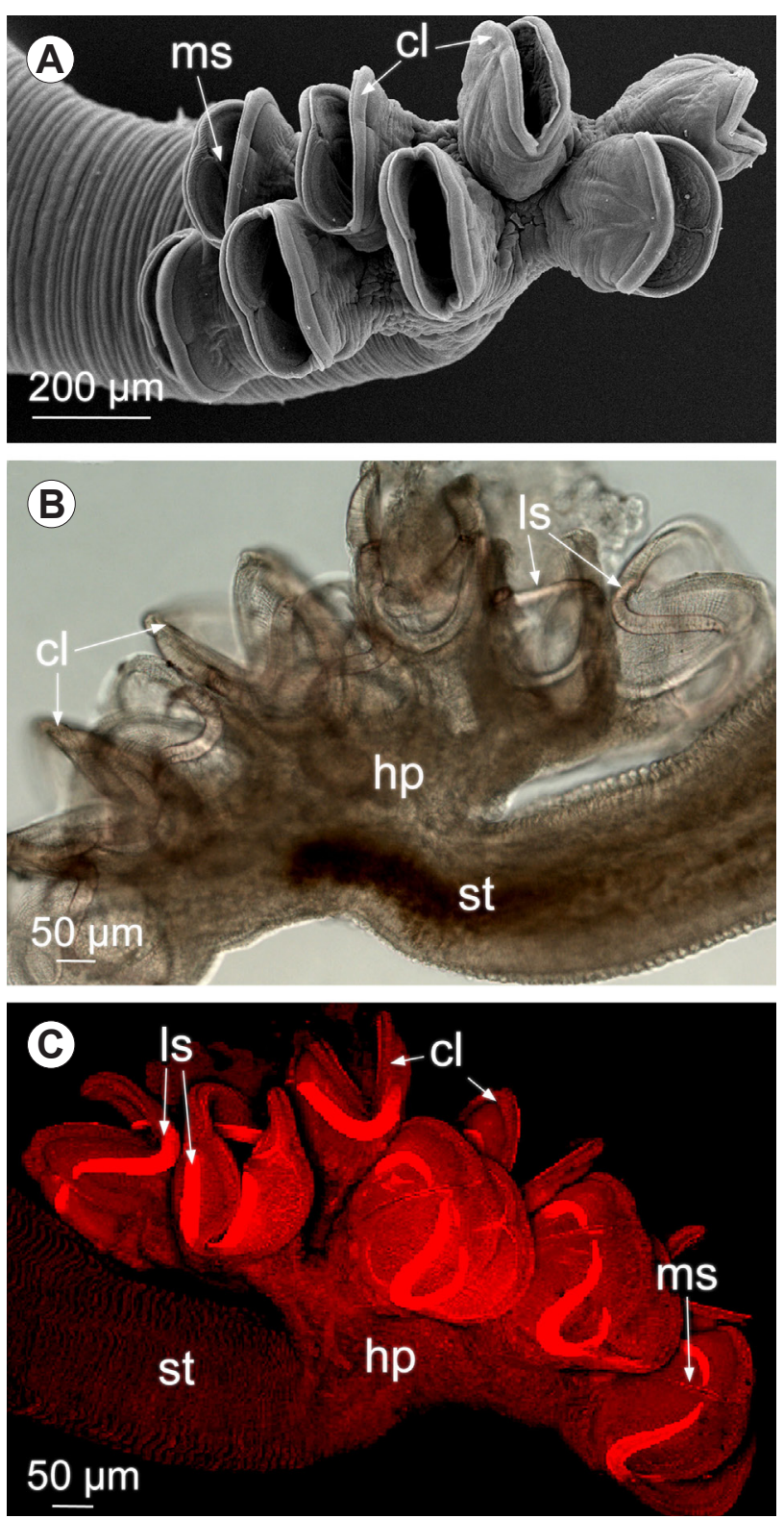

Fig. 2. The attachment organs of Chimaericola leptogaster (Leuckart, 1830). A - Scanning electron microscope; B - light microscopy, C - Scanning electron microscope, TRITC-phalloidin staining of musculature. Abbreviations: cl - clamps; hp haptor; ls - lateral sclerites; ms - median sclerite; st - stalk.

(Sigma, USA) (PBS-T) according to the method described by Coons et al. (1955). The fixed worms were placed in $10 \%$ sucrose (Helicon, Moscow, Russia) solution in PBS for a week, then embedded in Tissue-Tek (USA), frozen at $-18^{\circ} \mathrm{C}$ and sectionned longitudinally into $14-16 \mu \mathrm{m}$ slices with Shandon Termo Cryomatrix cryostat (Termoelectron Corporation, San Diego, CA, USA). The sections were collected on poly-L-lysin coated slides (Polysin, Menzel-Glaser, Saarbrückener, Germany), dried for one hour at room temperature and then were used either for immunocytochemistry directly or stored at $-20^{\circ} \mathrm{C}$ for one-two weeks.

The whole mounts were incubated in primary antibodies for five days at $4{ }^{\circ} \mathrm{C}$ and then, after washing, the secondary goat anti-rabbit Alexa488 (Molecular Probes, Waltham, MA, USA) (1: 400) antibodies in PBS-T were added to the samples for five days at $4{ }^{\circ} \mathrm{C}$. The frozen sections were incubated with the primary antibody for two days and then with the secondary antibodies for three hours. Controls included: 1) the omission of the primary antibody and 2) the substitution of primary antibody with non-immune rabbit serum.

\section{Staining musculature with TRITC-conjugated phalloidin}

In order to study the relationship between the patterns of the 5-HT-immunopositive (-IP) nerve elements and the musculature, staining with TRITC-conjugated phalloidin (Sigma, USA, $1: 200)$ was performed according to the method described by Wahlberg (1998). The duration of phalloidin staining was $6-12$ hours.

\section{Confocal laser scanning and fluorescent microscopy}

Samples stained with anti-5HT and TRITC-labelled phalloidin were examined by fluorescent microscopy on a Leica DM 1000 (from A.N. Severtsov Institute of Ecology and Evolution of RAS, Centre of Parasitology, Pushchino, Russia) and Leica DM6000 fluorescent, as well as with a Leica TCS SP5 (Berlin, Germany) confocal scanning laser microscope (from Institute of Cell Biophysics of RAS, Pushchino, Russia). Images presented are either the optical sections (snapshots) or the maximal projections obtained during CLSM scanning of investigated samples through $24-78 \mu \mathrm{m}$ (occasionally up to $142 \mu \mathrm{m}$ ). Images from the fluorescent microscope were taken with a digital camera DC300F (Leica, Berlin, Germany). For Alexa488, a fluorescent filter I3 with the excitation spectrum of $450-490 \mathrm{~nm}$ and the emission spectrum of $515 \mathrm{~nm}$ was used, while tetramethylrhodamine isothiocyanate (TRITC) was detected with a N2.1 filter (excitation spectrum of 515-560 nm; emission spectrum of $590 \mathrm{~nm}$ ). The images were saved in TIFF format.

\section{Scanning electron microscopy}

For SEM observations, the live worms were fixed using 5\% glutaraldehyde in $0.1 \mathrm{M}$ sodium cacodylate buffer $(\mathrm{pH}$ 7.2) for six days at $5^{\circ} \mathrm{C}$. The fixed worms were dehydrated in a graded ethanol series, with a final change in absolute ethanol and then critical-point-dried with liquid $\mathrm{CO}_{2}$. The specimens were mounted on stubs, sputter-coated with gold-palladium and examined using a JEOL-JSM-6510LV scanning electron microscope operating at $30 \mathrm{kV}$ (I.D. Papanin Institute for Biology of Inland Waters of RAS, Borok, Russia).

\section{RESULTS}

\section{The body surface of Chimaericola leptogaster - scanning electron microscopy}

The body of adult $C$. leptogaster is divided into three parts: the main body, the elongate narrow stalk and the haptor at its extreme posterior end (Figs. 1A, 2A). The haptor bears four pairs of bowl-like, symmetrically arranged clamps on short peduncles (Fig. 2A). Under SEM, shallow and transverse ridges are apparent on the whole surface of the body (Fig. 1A,H). The anterior end of $C$. leptogaster bears a mouth opening on the ventral body side (Fig. 1A,B); anterior to it the preoral, narrow, horizontal crevice can be observed (Fig. 1A,B).

The common genital pore is situated midventrally, at a distance of about $350 \mu \mathrm{m}$ from the posterior end of the buc- 


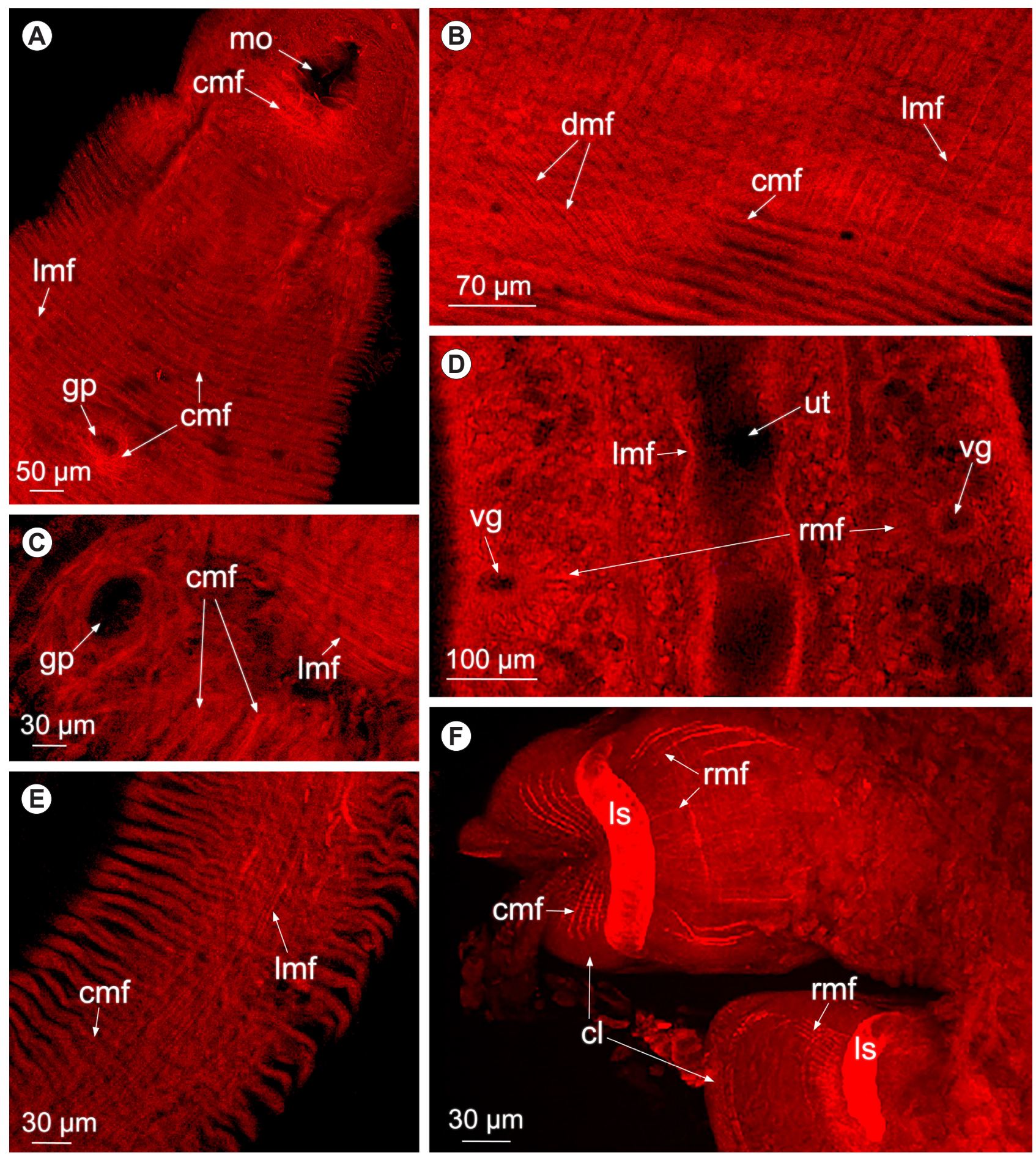

Fig. 3. Musculature of Chimaericola leptogaster (Leuckart, 1830) stained with TRITC-phalloidin (in red), CLSM (A, C, D, E - optical sections, B, F - maximal projections). A, C, E- circular, longitudinal and diagonal muscle fibres (arrows) in anterior (A, C), median (B) and posterior (E) body parts; (A, C - circular muscle fibres surrounding a genital pore); D - radial musculature of two vaginae and longitudinal muscle fibres of the uterus; $\mathbf{E}$ - longitudinal and circular muscle fibres in the stalk; $\mathbf{F}$ - circular and radial muscle fibres in the clamps of the haptor; note an intensive staining with TRITC-phalloidin in lateral sclerites of each clamp. Abbreviations: $\mathrm{cl}$ - clamps; cmf - circular muscle fibres; dmf - diagonal muscle fibres; gp - genital pore; lmf - longitudinal muscle fibres; ls - lateral sclerites; mo - mouth opening; rmf - radial muscle fibres; ut - uterus; vg - vagina.

cal cavity (Fig. 1A). Further posteriorly, two vaginal pores occur ventrosublaterally, one on either side of the body, at a distance of about $800 \mu \mathrm{m}$ from the posterior end of the buccal cavity and separated from each other by about $600 \mu \mathrm{m}$ (Fig. 1A). The anteroventral body surface around the mouth opening and preoral crevice are richly supplied with uniciliated (rarely biciliated and papillate multiciliated) and non-ciliated (papillae) sensory endings (Fig. 1C,F). Furthermore, uniciliated and non-ciliated sensory endings are scattered around the genital atrium and vaginae, as well as on the ventral body surface between the mouth opening and genital pores (Fig. 1A,B,H). 

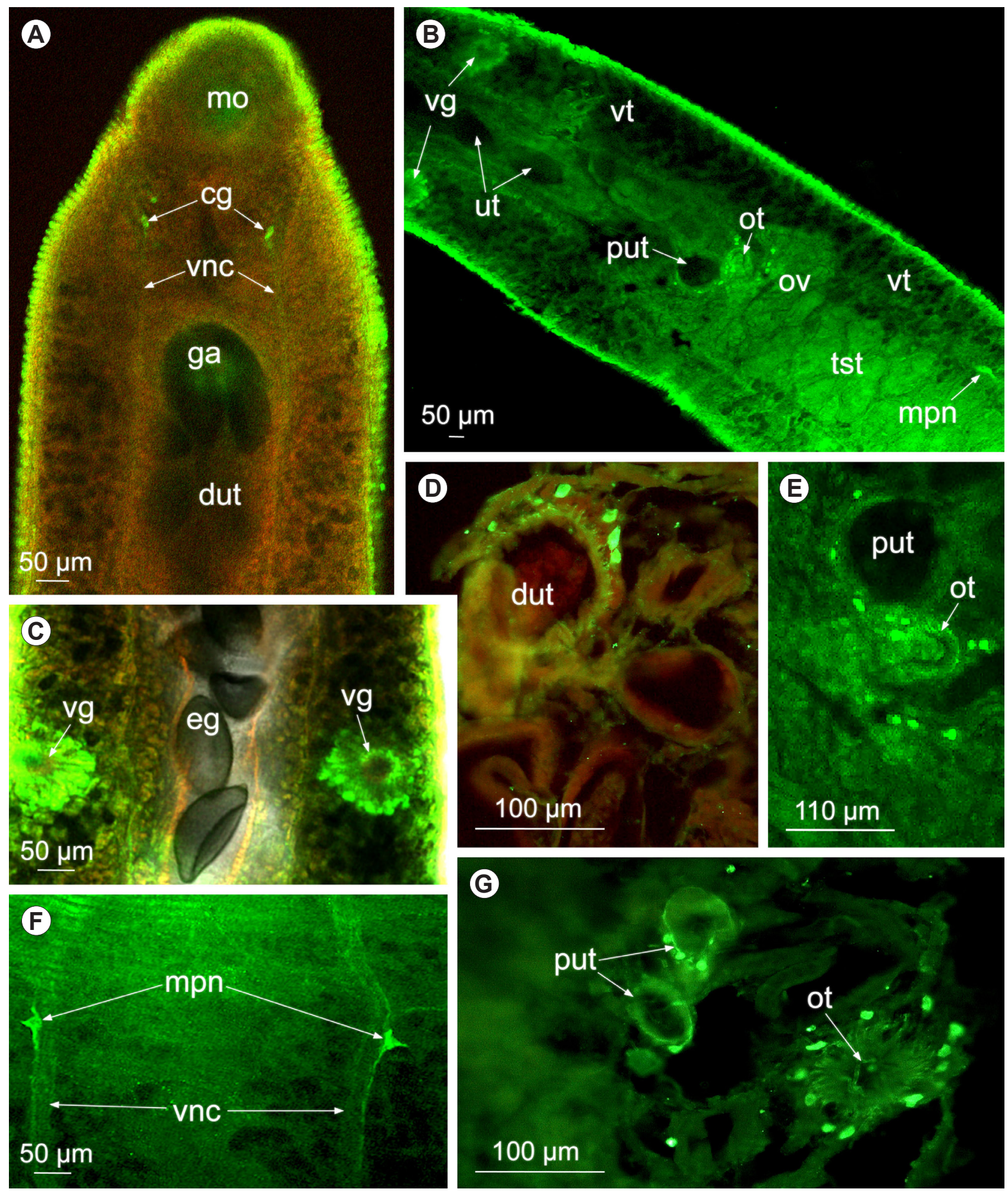

Fig. 4. 5-HT-immunopositive staining (in green) in Chimaericola leptogaster (Leuckart, 1830) tissue; musculature (in red) stained by TRITC-phalloidin, CLSM (A-C, E, F - whole mounts) and fluorescent (D, G - sections) microscopy. A - an anterior body end with cephalic nerve ganglia (arrows) and a pair of longitudinal ventral nerve cords (arrows), mouth opening and distal uterus; $\mathbf{B}$ - a middle body part (more detailed on c and f), with a pair of vaginae (arrows), and major compartments of female - uterus (arrow), vitellarium, ootype (arrow), proximal uterus, ovary, and male reproductive system - testes; $\mathbf{C}-5$-HT-IP staining among the musculature of vaginae (long arrows); the uterus cointains eggs; D - 5-HT-IP neurons surrounding the reproductive duct of the distal uterus; E - 5-HT-IP elements in the vicinity of the ootype and proximal part of the uterus; $\mathbf{F}$ - a pair of large multipolar 5-HT-IP neurons (long arrows) in the middle part of the body within the ventral nerve cords (arrows); G - 5-HT-IP neurons surrounding the ducts of proximal uterus (arrows), and about twelve of 5-HT-IP neurons around the ootype (arrow). Abbreviations: cg - cephalic ganglia; dut - distal uterus; eg - egg; ga - genital atrium; mo - mouth opening; mpn - main paired neurons; ot - ootype; ov - ovary; put - posterior uterus; tst - testes; ut - uterus; vg - vagina; vnc - ventral nerve cord; vt - vitelline follicles. 

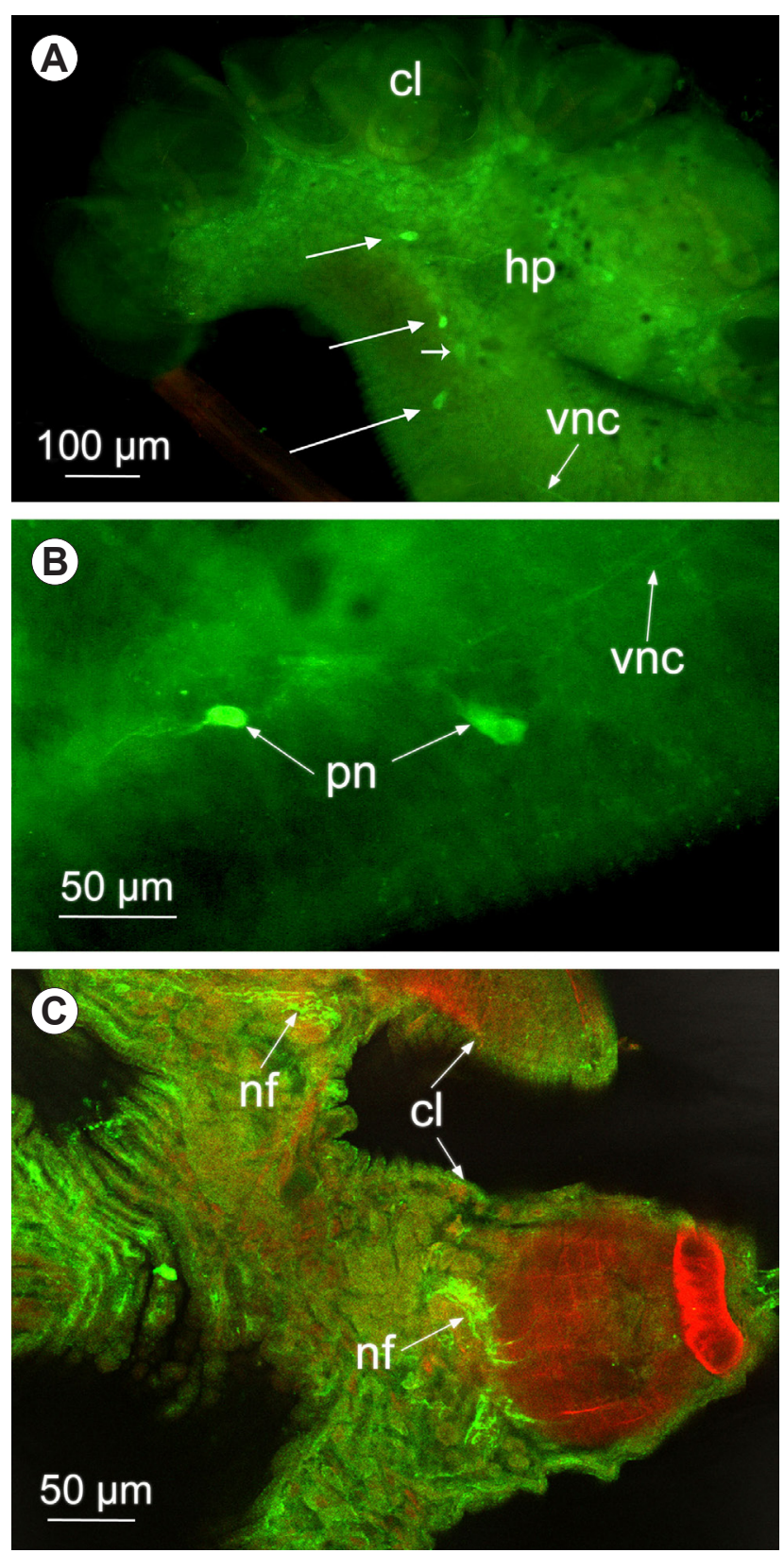

Fig. 5. The serotonin-immunopositive neurons and fibres (in green) innervating the attachment organs of Chimaericola leptogaster (Leuckart, 1830); musculature (in red) stained by TRITC-phalloidin (A, B - fluorescent and C - CLSM images).

A, B - the 5-HT-IP neurons in the base of the stalk (arrows); $\mathbf{C}$ - the 5-HT-IP nerve fibres in the base of the clamps (arrows) Abbreviations: cl - clamps; hp - haptor; nf - nerve fibres; $\mathrm{pn}$ posterior neurons; vnc - ventral nerve cord.

Under SEM we can identify some features of the sensory structure: uniciliated sensory endings with short (about $0.9 \mu \mathrm{m}$ long), medium (about $2 \mu \mathrm{m}$ long) and long (about $4 \mu \mathrm{m}$ long) cilia (Fig. 1C,E,G); biciliated sensory endings with short cilia (Fig. 1F); papillate bulb containing six to seven short (about $0.5-0.7 \mu \mathrm{m}$ long) cilia in one bulb (Fig. 1D) and papillae with non-ciliated sensory endings (Fig. 1H). Each of the eight haptoral clamps of C. leptogaster is a bowl-shaped structure that possesses a single median and paired lateral sclerites (Fig. 2A-C). The lateral sclerites are situated deep within the muscle blocks of the clamp (Fig. 2B,C) and have a similar internal structure to the basement lamina surrounding the muscle blocks of each clamp.

\section{Musculature morphology identified by TRITC- phalloidin staining}

The histochemical staining of the tissue of C. leptogaster with fluorescently-labelled phalloidin revealed the presence of well-developed longitudinal, circular and diagonal muscles that run along the whole body (Fig. 3A,B), including the stalk - the narrow region before the haptor (Fig. 3E). In the middle part of the body, the thickness of the circular muscle layer was estimated to be 15-20 $\mu \mathrm{m}$ (Fig. 3B). Around the genital pore (Fig. 3A,C), circular muscle fibres were observed. In the two vaginae the radial muscles fibres were also intensively stained (Fig. 3D).

Our investigation also demonstrates the presence of well-developed muscle fibres in the clamps of the adhesive organ of $C$. leptogaster. Besides the presence of longitudinal and circular muscles in each clamp, the muscle blocks of each clamp consist of radially distributed muscle fibres (Fig. 3F). Intensive staining with phalloidin was revealed in the muscular sheath of the lateral sclerites (Fig. 2C).

\section{Serotonin-(5-HT)-immunoreactivity in central nervous system (CNS)}

5-HT-IP cells $(17 \times 27 \mu \mathrm{m})$, two on each side, were observed at a distance of $200 \mu \mathrm{m}$ from the mouth opening (Fig. 4A). Two longitudinal (ventral) 5-HT-IP nerve cords run in parallel along the body (Fig. 4A,F). In the middle of the body (behind the vaginal openings), there is a pair of large $(25-27 \mu \mathrm{m})$ multipolar serotonin-IP nerve cells, carrying at least three long processes; each of these cells was found in the ventral nerve cords (Fig. 4B,F).

\section{5-HT-immunopositive staining in the reproductive system}

Immunocytochemical staining of the whole mounts and frozen sections of $C$. leptogaster shows the presence of a cluster of small $(5 \mu \mathrm{m})$ serotoninergic nerve cells surrounding the ootype and proximal part of the uterus (Fig. 4D,E,G). A positive serotonin staining was also observed among the muscles comprising the paired vaginae, genital pore and other ducts of the female and male reproductive system (Fig. 4A,B,C).

\section{5-HT-immunopositive nerve elements in attachment organs}

In the posterior body region, in close proximity to the haptor, there are two pairs of 5-HT-IP nerve cells (15$20 \mu \mathrm{m}$ ), located alongside of the nerve cords extended in the posterior body part. These cells were located approximately $80-100 \mu \mathrm{m}$ from each other (Fig. 5A,B). In the attachment organ of $C$. leptogaster a plexus comprised of 5-HT-IP nerve fibres was found. The 5-HT-IP nerve fibres were found in the clamps themselves (Fig. 5C). The distri- 


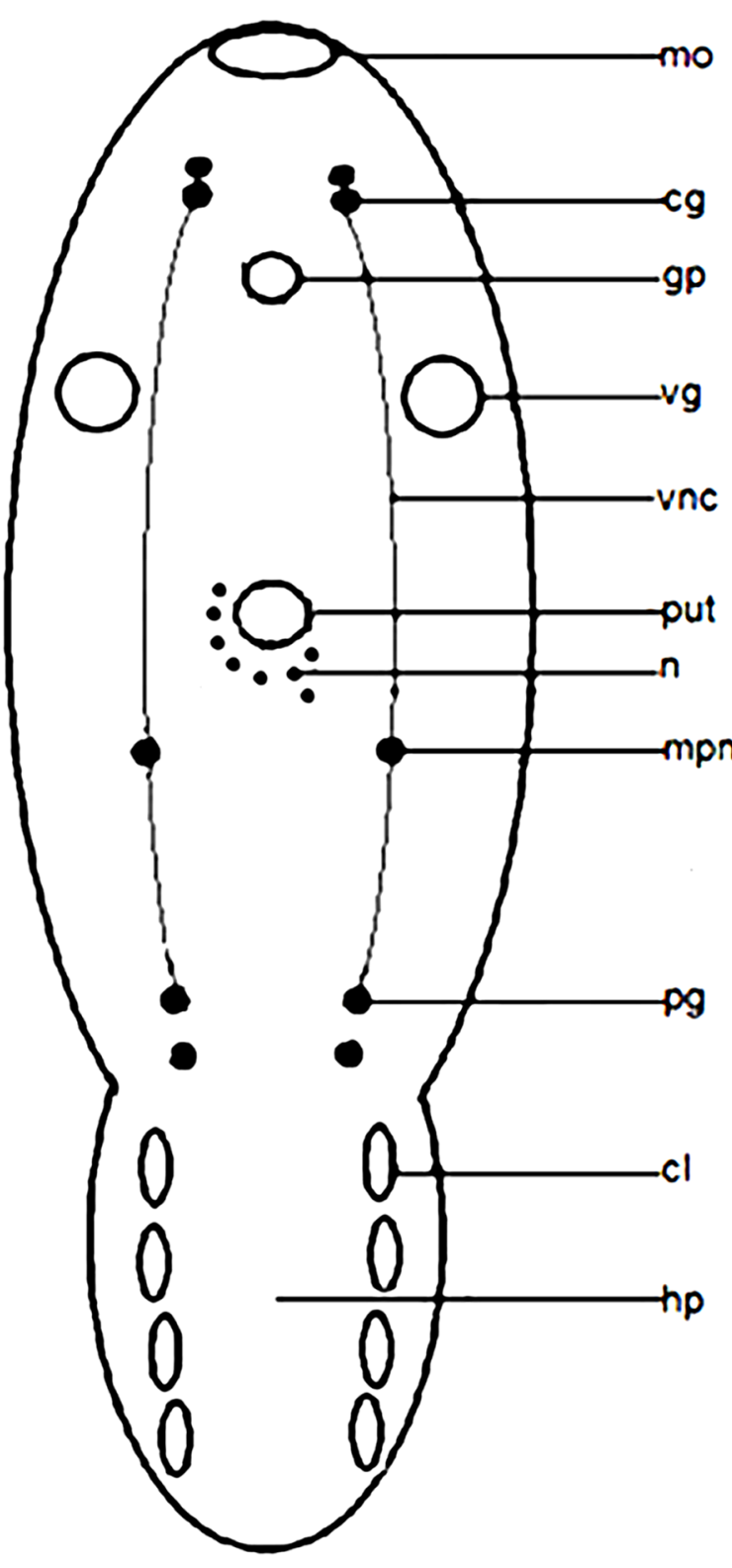

Fig. 6. Schematic drawing of serotoninergic nerve elements distribution in Chimaericola leptogaster (Leuckart, 1830).

Abbreviations: cl - clamps; cg - cephalic ganglia; gp - genital pore; hp - haptor; mo - mouth opening; mpn - main paired neurons; $\mathrm{n}$ - neurons; pg - posterior ganglia; put -posterior uterus; vg - vagina; vnc - ventral nerve cord.

bution of serotonin-immunopositive elements in the NS of C. leptogaster is shown in Fig. 6.

\section{DISCUSSION}

Muscle system. In the present study, data are presented on the morphology of both the neuromuscular system and the attachment organs of Chimaericola leptogaster. This monogenean fixes itself tightly to the gills of its host by an attachment organ, an adhesive disc or a haptor, situated at the posterior end of the body. The adhesive disc of C. leptogaster is comprised of eight specialised muscular structures: the clamps, each of which contains two lateral and one median sclerites (Brinkmann 1942, Bychowsky 1957, Poddubnaya et al. 2014).
Information on muscular and nerve elements in C. leptogaster has so far been absent. Our study revealed the presence of well-developed circular and longitudinal muscles, comprising the musculature of the body of C. leptogaster. We also observed diagonal muscle fibres in the body wall, which have been described in Gyrodactylus rysavyi (Ergens, 1973) and Microgyrodatylus congolensis (Prudhoe, 1957) (see Arafa et al. 2007, El-Naggar 2007). In our study, muscle fibres intensively stained with phalloidin were observed around the mouth opening of $C$. leptogaster as well as in the attachment organ and around the reproductive pore and vaginae. The data obtained correspond to those for other monogenean species G. rysavyi, M. congolensis and Paradiplozoon homoion (Bychowsky et Nagibina, 1959), in which muscle fibres were detected in the attachment organs, as well as in the ducts of the digestive and reproductive systems (Arafa et al. 2007, El-Naggar et al. 2007, Hodová et al. 2018).

Serotoninergic nerve elements in CNS. The data obtained on the existence and localisation of serotoninergic nerve elements in the nervous system of $C$. leptogaster are generally in agreement with the information available for other monogeneans (Zurawski et al. 2001, Rastogi et al. 2012, Petrov et al. 2017).

Our study revealed positive immunostaining to serotonin in two symmetric nervous structures situated in the anterior body end of $C$. leptogaster, which most probably correspond to the CNS compartments, particularly to the cephalic ganglia, described in other monogeneans (Cable et al. 1996, Halton et al. 1998). From the cephalic ganglia, a pair of clearly visible longitudinal nerve cords run along the body trunk towards the posterior body end. According to the literature, monogeneans exhibit three pairs of longitudinal nerve cords that run from the brain ganglia; of these, the ventral nerve cords are most pronounced (Arafa et al. 2007, El-Naggar et al. 2007). In our study we were able to identify only the ventral nerve cords stained with anti-serotonin. Therefore, that is why further investigations are necessary.

Innervation of the reproductive system organs Amongst monogeneans, a complex hermaphroditic reproductive system includes a number of male organs - testes, vas deferens, seminal vesicle, and cirrus, and female organs - ovary, oviduct, seminal receptacle, ootype and Mehlis gland and uterus together with vitellarium, vitelline ducts and associated reservoirs (Maule et al. 1990b).

The innervation of different reproductive organs by serotoninergic nerves has been shown in the monogenean species of Entobdella soleae (Van Beneden et Hess, 1863), G. rysavyi and Polystoma integerrimum (see McKay et al. 1991, Marks et al. 1994, Arafa et al. 2007). In Diclidophora merlangi (Kuhn, in Nordmann, 1832) serotoninergic cells and fibres were only found in the male parts of the reproductive system - cirrus, seminal vesicle and vas deferens (Maule et al. 1990b). The 5-HT-IP fibres were observed in the copulatory organ, the seminal vesicle and the testis of M. congolensis (El-Naggar et al. 2007). In the work of Zurawski et al. (2001) the serotoninergic nerve elements 
were not identified in the ootype of Eudiplozoon nipponicum (Goto, 1891).

The present data revealed the intensive innervation of the ducts of the reproductive tract of $C$. leptogaster, including the egg-forming complex ducts (ootype and proximal uterus) by 5-HT-IP nerve cells and fibres. Stained serotonin-IP elements were identified in paired vaginae and around the metraterm of the distal uterus. It should be noted that ultrastructural studies on female ducts of C. leptogaster show that the ootype and proximal uterus are surrounded by numerous nerve cells, forming an extensive neuronal plexus of the cell bodies along the duct wall (Poddubnaya et al. 2017 and unpublished data). Close association of the nerve cells with the neuronal plexus and numerous receptors has been described around vaginal pores and along the distal portion of the vaginal tubes of $C$. leptogaster by Poddubnaya et al. (2013).

Serotonin in the innervation of the attachment organs. According to Cable et al. (1996), two pairs of serotoninergic neurons have been found in the haptor of the monogenean Discocotyle sagittata (Leuckart, 1842). The innervation of the attachment organs by serotoninergic nerve fibres was also observed in E. nipponicum, $P$. integerrimum, P. homoion and M. congolensis (McKay et al. 1991, Zurawski et al. 2001, El-Naggar et al. 2007, Hodová et al. 2018).

The results of our study indicated the presence of several 5-HT-IP nerve cells that were located alongside nerve cords in close proximity to the haptor of C. leptogaster. In the attachment organs of this monogenean the plexus comprised of 5-HT-IP nerve fibres has been found.

In the present study, new immunocytochemical data on the NS and the muscular systems of C. leptogaster were obtained. The data indicate the presence of serotoninergic neurons and fibres in the central and peripheral compartments of the monogenean NS. The distribution of serotoninergic elements in C. leptogaster generally correspond- ed to the previously described orthogonal NS pattern that most monogenean species are known for. At the same time, some differences were found. Thus, the number of serotoninergic neurons in cephalic ganglia and nerve cords were less abundant than in other monogenean species. Only one pair of longitudinal nerve cords was observed. The central nerve commissure, which usually connects paired cephalic ganglia as well as transversal nerve commissures connecting the lateral nerve cords, has not yet been identified.

The relatively scarce amount of serotonin-IP nerve element detected in the central compartment of C. leptogaster NS can be explained by the fact that in monogeneans the serotoninergic neurons comprise a minor part of the central nervous system compared to the cholinergic and the peptidergic components. Comparative analysis indicates that serotoninergic elements in the nervous system of $C$. leptogaster were less predominant than in other investigated monogenean species. Because of its relict nature and because of an insufficient number of specimens available for staining, we could not rule out the fact that not all of the serotoninergic compounds were disclosed in C. leptogaster, which means that additional research is required. The data obtained in the study of the serotoninergic innervations of genital ducts and the attachment organ of $C$. leptogaster confirm the important role of serotonin in the regulation of the reproductive processes and muscle contractions of flatworms.

Acknowledgements. The authors would like to thank Willy Hemmingsen, University of Tromsø (Norway) and the staff of the RV 'Johan Ruud' of Tromsø University for their help with fishing and collecting material. The authors are grateful to the staff of the Centre of Electron Microscopy, I.D. Papanin Institute for Biology of Inland Waters, Russian Academy of Sciences, Borok, for their technical assistance in SEM investigations. This work was partly supported by the Russian Foundation for Basic Research (grant No.18-04-00349a to N. D. K.) and by program No. AAAA-A18-118012690100-5 of the Russian Academy of Scinces Institute for Biology of Inland Waters.

\section{REFERENCES}

Arafa S.Z., El-Naggar M.M., El-Abbassy S.A., Stewart M.T., Halton D.W. 2007: Neuromusculature of Gyrodactylus rysavyi, a monogenean gill and skin parasite of the catfish Clarias gariepinus. Parasitol. Int. 56: 297-307.

BRINKMANN A.JR. 1942. On "Octobothrium" leptogaster F.S Leuckart. Göteborgs Kungl. Vet. Vitt. Samhs. Handl. Ser B 2: 3-29.

Bychowsky B.E. 1957: [Monogenetic Trematodes, their Systematics and Phylogeny]. Izdatel'stvo Academii Nauk SSSR, Moscow, 509 pp. (In Russian.)

Cable J., Marks N.J., Halton D.W., Shaw C., Johnston C.F., Tinsley R.C., GannicotT A.M. 1996: Cholinergic, serotoninergic and peptidergic components of the nervous system of Discocotyle sagittata (Monogenea: Polyopisthocotylea). Int. J. Parasitol. 26: 1357-1367.

Coons A.H., Leduc E.H., Connolly J.M. 1955: Studies on antibody production. I. A method for the histochemical demonstration of specific antibody and its application to a study for the hyperimmune rabbit. J. Exper. Med. 102: 49-60.
El-Naggar M.M., Arafa S.Z., El-Abbassy S.A., Stewart M.T., Halton D.W. 2004: Neuromusculature of Macrogyrodactylus clarii, a monogenean gill parasite of the Nile catfish Clarias gariepinus in Egypt. Parasitol. Res. 94: 163-175.

El-Naggar M.M., Arafa S.Z., El-Abbassy S.A., Stewart M.T., Halton D.W. 2007: Neuromusculature of Macrogyrodactylus congolensis, a monogenean skin parasite of the Nile catfish Clarias gariepinus. Parasitol. Res. 100: 265-279.

Halton D.W. , Jennings J.B. 1964: Demonstration of the nervous system in the monogenetic trematode Diplozoon paradoxum Nordmann by the indoxyl acetate method for esterases. Nature 202: $510-511$.

Halton D.W., Maule A.G., Johnston C.F, Fairweather I. 1987: Occurrence of 5-hydroxytryptamine (serotonin) in the nervous system of a monogenean, Diclidophora merlangi. Parasitol. Res. 74: 151-154.

Halton D.W., Maule A.G., Mair G.R., Shaw Ch. 1998: Monogenean neuromusculature: some structural and functional correlates. Int. J. Parasitol. 28: 1609-1623. 
Halton D.W. AND MorRis G.P. 1969: Occurrence of cholinesterase and ciliated sensory structures in the fish gill fluke $D i$ clidophora merlangi (Trematoda: Monogenea). Z. Parasitenkd. 33: $21-30$.

Hodová I., Sonnek R., Gelnar M., Valigurová A. 2018: Architecture of Paradiplozoon homoion: a diplozoid monogenean exhibiting highly developed equipment for ectoparasitism. PLoS ONE 13: e0192285.

Inoue J.G., Miya M., Lam K., Tay B.H., Danks J.A., Bell J., Walker T.I., Venkatesh B. 2010: Evolutionary origin and phylogeny of the modern holocephalans (Chondrichthyes: Chimaeriformes): a mitogenomic perspective. Mol. Biol. Evol. 27: 2576-2586.

Marks N.J., Halton D.W., Kearn G.C., Shaw C., Johnston C.F. 1994: 5-hydroxytryptamine-immunoreactivity in the monogenean parasite, Entobdella soleae. Int. J. Parasitol. 24: 1011-1018.

Maule A.G., Halton D.W., Allen J.M., Fairweather I. 1989: Studies of motility in vitro of an ectoparasitic monogenean, Diclidophora merlangi. Parasitology 98: 85-93.

Maule A.G., Halton D.W., Johnston C.F., Shaw C., FairWEATHER I. 1990a: The serotoninergic, cholinergic and peptidergic components of the nervous system in the monogenean parasite, Diclidophora merlangi: a cytochemical study. Parasitology 100: 255-273.

Maule A.G., Halton D.W., Johnston C.F., Shaw C., FairWEATHER I. 1990b: A cytochemical study of the serotoninergic, cholinergic and peptidergic components of the reproductive system in the monogenean parasite, Diclidophora merlangi. Parasitol. Res. 76: 409-419.

McKay D.M., Halton D.W., Maule A.G., Jonston C.F., Shaw C., Fairweather I. 1991: Putative neurotransmitters in two monogeneans. Helminthologia 28: 75-81.

Petrov A., Gerasev P.I., Popyuk M.P., Dmitrieva E.V. 2016 Haptoral neuromusculature in two species of Dactylogyrus Diesing, 1850 (Monogenea: Dactylogyridae). Syst. Parasitol. 93: 337-354.

Petrov A.A., Dmitrieva E.V., Popyuk M.P., Gerasev P.I., Petrov S.A. 2017: Musculoskeletal and nervous systems of the attachment organ in three species of Diplectanum (Monogenea: Dactylogyroidea). Folia Parasitol. 64: 022.

Poddubnaya L.G., Hemmingsen W., Gibson D.I. 2013: Ultrastructural characteristics of the vaginae of the basal monogenean Chimaericola leptogaster (Leuckart, 1830). Parasitol. Res. 112: 4053-4064

Poddubnaya L.G., Hemmingsen W., Gibson D.I. 2014: Clamp ultrastructure of the basal monogenean Chimaericola leptogaster (Leuckart, 1830) (Polyopisthocotylea: Chimaericolidae). Parasitol. Res. 113: 4023-4032.

Poddubnaya L.G., Hemmingsen W., Gibson D.I. 2017. The unique uterine structure of the basal monogenean Chimaericola leptogaster (Monogenea: Polyopisthocotylea), an ectoparasite of the relictual holocephalan fish Chimaera monstrosa. Parasitol. Res. 116: 2695-2705.

Raikova O.I., Kotikova E.A., Frolova T.A. 2017: [Nervous system and musculature of the parasitic turbellarian Notentora ivanovi (Plathelminthes, Fecampiida)]. Dokl. Biol. Sci. 475: 593-595. (In Russian.)

Rastogi P., Mishra D., Singh J., Rani K. 2012: Neuroanatomy of Cornudescoides kulkarnii n. sp., a gill parasite of Mystus vittatus in Meerut (UP). J. Parasit. Dis. 36: 61-65.

Terenina N.B. And Gustafsson M.K.S. (ED.) 2003: [Neurotransmitters in helminths]. Nauka, Moscow, 177 pp. (In Russian.)

Timofeeva T.A. And Kotikova E.A. 1995: [Topographical peculiarities of monogenean nervous systems]. Parazitologiya 29: 127-139. (In Russian.)

Venkatanarsaiah J. and Kulkarni T. 1980. Studies on the monogenetic trematodes of Andhra Pradesh: contribution to our knowledge of Tympanocirrus Tripathi, 1959 with a description of T. trygoni n. sp. and a rediscription of Onchiodiscus pterodiscoides Kulkarni, 1969. Riv Parassitol. 41: 85-91.

Wahlberg M.H. 1998: The distribution of F-actin during the development of Diphyllobothrium dendriticum (Cestoda). Cell Tiss. Res. 291: 561-70.

Zurawski T.H., Mousley A., Mairb G.R., Brennan G.P., Maule A.G., Gelnar M., Halton D.W. 2001: Immunomicroscopical observations on the nervous system of adult Eudiplozoon nipponicum (Monogenea: Diplozoidae). Int. J. Parasitol. 31: 783-792.

Cite this article as: Mochalova N.V., Terenina N.B., Poddubnaya L.G., Yashin V.A., Kuchin A.V., Kreshchenko N.D. 2019: First evidence of serotoninergic components in the nervous system of the monogenean Chimaericola leptogaster (Chimaericolidae, Polyopisthocotylea), gill parasite of the relict holocephalan fish. Folia Parasitol. 66: 008. 